Revue d'histoire de l'Amérique française

RAS REVUE D.HISTOIRE DE L'AMÉRIQUE FRANÇAISE

\title{
La question des vivres au Canada au cours de l'hiver 1757-1758
}

\section{Gilles Archambault}

Volume 21, numéro 1, juin 1967

URI : https://id.erudit.org/iderudit/302643ar

DOI : https://doi.org/10.7202/302643ar

Aller au sommaire du numéro

Éditeur(s)

Institut d'histoire de l'Amérique française

ISSN

0035-2357 (imprimé)

1492-1383 (numérique)

Découvrir la revue

Citer cet article

Archambault, G. (1967). La question des vivres au Canada au cours de l'hiver 1757-1758. Revue d'histoire de l'Amérique française, 21(1), 16-50.

https://doi.org/10.7202/302643ar d'utilisation que vous pouvez consulter en ligne.

https://apropos.erudit.org/fr/usagers/politique-dutilisation/ 


\section{LA QUESTION DES VIVRES AU CANADA AU COURS DE L'HIVER 1757-1758}

L'étude de cet aspect particulier de la vie coloniale canadienne sous le régime français comporte un double danger. Il y a tout d'abord, le risque d'isoler ce phénomène en omettant de l'insérer à l'intérieur d'un ensemble dynamique de forces indissociables les unes des autres. Ainsi, par exemple, une insistance marquée sur quelques secteurs de l'économie comme la production agricole coloniale ou les importations, peut fausser la réalité historique. Elle valorise telle série de causes au détriment de telle autre. L'historien doit éviter ce clivage arbitraire et situer son étude dans une perspective plus vaste d'interaction de facteurs. La question des vivres au Canada est inséparable non seulement des données économiques mais aussi des conditions géographiques et démographiques, des impératifs, politiques et militaires et des courants psychologiques et sociaux. Dans une étude du problème de l'alimentation durant l'hiver 1757-1758, l'historien doit tenir compte de cette aire horizontale d'interdépendance de facteurs pour ce bref moment historique.

Parmi ces facteurs déterminants, certains sont inhérents et propres à la situation, mais il y en a d'autres qui ont un caractère de permanence et qui ne sont nullement accidentels. Et c'est ici que réside un autre danger. Une connaissance objective et une compréhension véritable de la situation exigent que cette courte période historique soit replacée dans une longue courbe d'évolution, dans un ensemble structural, vertical ou diachronique. Impossible d'avoir une idée exacte sur la question des vivres au Canada durant l'hiver 1757-1758 sans situer ce problème à l'intérieur du phénomène de la colonisation française en Nouvelle-France et $d u$ vaste et lent mouvement de 
l'expansion colonisatrice de la civilisation occidentale en Amérique. C'est dans cette optique géohistorique que seront envisagées l'analyse des principales données du problème ainsi que la recherche de leurs causes explicatrices.

\section{1 - LES DONNÉES DU PROBLEME}

En premier lieu, une connaissance de la situation elle-même c'est-à-dire des événements qui se sont déroulés pendant l'hiver 1757-1758, est nécessaire. Ces faits semblent se résumer à peu près à ceci: diminution progressive de la quantité de vivres par habitant; inégalité de cette diminution entre les différentes classes de la société; état de crise chez les classes les plus défavorisées.

\section{DISETTE ET RATIONNEMENT}

Plusieurs documents sont unanimes à décrire la disette qui a sévi au Canada durant l'hiver 1757-1758. La farine et la viande de bœuf, qui constituaient à l'époque la base essentielle de tout régime alimentaire, devinrent de plus en plus difficile à trouver. L'administration coloniale dut intervenir et la population fut soumise à la ration quotidienne. La quantité et la qualité de celle-ci diminuèrent progressivement. La portion journalière de pain passa d'une demi-livre à quatre onces, de quatre onces à deux onces et même à rien du tout tandis qu'à certains moments, on dut remplacer la farine par le riz, et le bœuf, par le cheval, la morue ou le lard.

La situation qui a prévalu est le prolongement de ce qui avait commencé au cours des saisons précédentes. Dès le printemps et l'été 1757 , la pénurie de vivres s'était fait sentir. Au mois de juillet, Montcalm écrivait: "La disette où nous sommes de vivres augmente chaque jour. Québec souffre beaucoup et, si sous quinze jours, nos bâtiments de transports n'arrivent, nous seront fort embarrassés". ${ }^{1}$ L'inévitable se produisit. Aucun

1 Vaudreuil à Lévis, 19 juillet 1757, Collection des manuscrits du Chevalier de Lévis, R. H. Casgrain, éditeur (désormais cité Casgrain), 8: 42 . 
bateau ne se présenta et les dirigeants furent contraints de prendre les mesures nécessaires pour diminuer le risque de disette totale. Il fallait rationner les troupes et la population coloniale. Chaque habitant avait droit à un quarteron de pain par jour. Quant aux militaires, le 31 août, Vaudreuil écrivait à Lévis: "Je vous prie de faire continuer à ne donner aux troupes que la livre et demie de pain et un quarteron de lard ainsi que $\mathrm{M}$. le marquis de Montcalm m'a dit l'avoir ordonné avant son départ." 2

On croyait que ces mesures ne seraient que temporaires. On espérait que, dès septembre, grâce à la récolte et à l'arrivée de vaisseaux métropolitains, la menace d'une disette prolongée serait écartée. Mais tel ne fut pas le cas. Dans son journal, le 16 septembre 1757, Bougainville précise: "La disette était extrême. On continue à ne donner que quatre onces de pain par tête et l'on doute que cette ration puisse être tout l'hyver fournie sur ce pied." 3 Les craintes de Bougainville se justifièrent; à la rentrée des soldats dans les villes pour l'hiver, des mesures nouvelles de rationnement furent prises, non seulement, cette fois, sur le pain mais aussi sur les viandes. L'alimentation du soldat fut minutieusement réglementée:

M. Bigot ayant fait l'exposition de la situation de la colonie à $\mathbf{M}$. le marquis de Vaudreuil et à $\mathbf{M}$. le marquis de Montcalm, dans un conseil où on a appelé le sieur Péan et le munitionnaire général..., il a été arrêté qu'à commencer le ler novembre, la distribution des vivres se feroit au soldat pour huit jours, savoir: à raison d'une demi-livre de pain par jour et un quarteron de pois, six livres de bœuf et deux livres de morue pour huit jours, et qu'en décembre on commenceroit à donner du cheval.4

$\mathrm{Au}$ mois de décembre, on voulut soumettre la population coloniale à une épreuve de force: la priver de son quarteron de pain et l'obliger à manger du cheval. "Le 1er du mois de décem-

${ }^{2}$ Vaudreuil à Lévis, 31 août 1757, Casgrain, 8: 44.

3 Journal de Bougainville, 16 septembre 1757, Rapport de l'Archiviste de la province de Québec (désormais RAPQ), (1923-1924), 308-309.

4 Journal du Marquis de Montcalm, 14 octobre 1757, Casgrain, 7: 308. 
bre, l'on ôta entièrement le quarteron de pain que l'on distribuoit au peuple, et, à la place, on voulut faire donner moitié bœuf et moitié cheval, dont la livre de l'un et de l'autre ne seroit payée que six sols." ${ }^{5} \AA$ la suite de nombreuses protestations, on dut revenir au quarteron de pain.

Et, ce fut le long "hivernement" dans les privations et la misère. "Notre triste situation... n'a pas changé, le peuple perit de Misere les acadiens refugiés Ne Mangent depuis quatre mois que du cheval et de la Merluche sans Pain." ' En avril et mai, on assiste à une augmentation de la misère et à une autre réduction des vivres. "Misère grande: le peuple réduit à deux onces de pain. Au lieu de la livre de bœuf ou de cheval, 1/2 de lard et un quarteron de morue." 7 "Nulles nouvelles de bâtimens. La misère est si grande que quelques habitans sont réduits à vivre d'herbe [légumes]." 8 Ces quelques témoignages démontrent clairement la progression décroissante de la quantité et de la qualité des vivres pour chaque habitant. Daine résume la situation:

Rien de plus triste et de plus affligeant que la situation actuelle de la colonie. Après avoir passé une partie de l'automne et de l'hiver dernier à un quarteron de pain par jour pour chaque personne, nous sommes depuis six semaines réduits à deux onces. Toutes les ressources sont épuisées, et nous sommes à la veille d'essuyer la plus cruelle famine si les secours n'arrivent pas dans quinze jours. Les expressions me manquent pour vous décrire nos malheurs. Les animaux commencent à manquer; les bouchers ne peuvent pas fournir un quart du bœuf nécessaire pour la subsistance des habitants de la ville. ${ }^{9}$ 117-118.

5 Journal du Chevalier de Lévis, 1er décembre 1757, Casgrain, 1:

6 Lettres de Doreil, lettre du 26 février 1758, RAPQ (1944-1945), 120.

7 Journal de Bougainville, 3 mai 1758, RAPQ (1923-1924), 319.

8 Journal de Bougainville, 21 mai 1758, RAPQ (1923-1924), 321.

9 Daine au Ministre de la Marine, mai 1758, cité dans A. J. Malartic, Journal des campagnes au Canada de 1755 à 1760, 170. 
Un état de sous-alimentation produit, sur la condition physique de la population, des effets nocifs que vient accentuer le facteur climatique du froid. L'hiver 1757-1758 fut très rude. Le thermomètre descendit plusieurs fois à 28 degrés au-dessous de zéro. Des conditions alimentaires et météorologiques aussi défavorables à la santé engendrèrent une recrudescence de la maladie et accrurent les risques de mort. "Il est fort à craindre, écrit Doreil, qu'a la suitte d'une pareille misere nous n'ayons a essuier des maladies serieuses, la nouriture (sic) de cheval n'est pas trop naturelle." ${ }^{10}$ La population fut décimée par la petite vérole. "On n'ose pas dire que la petite vérole tue autant que la guerre ... mais au mois de novembre, Montcalm donne pour les hôpitaux de Québec de 2500 à 2600 malades sur lesquels un cinquième succombe. Et les hôpitaux de Québec ne reçoivent pas tous les picotés de la colonie." 11 S'il convient d'ajouter les 300 à 400 Acadiens morts de faim au cours de l'hiver, le nombre de décès s'élève à près de 1000. C'est un taux de mortalité très élevé pour la petite population de la région de Québec à cette époque; conséquences désastreuses pour une colonie qui avait un besoin considérable de maind'œuvre.

\section{INEGALITÉS SOCIALES}

Bien que cette disette eût pris des proportions assez importantes, il ne semble pas qu'elle aît atteint également toutes les classes de la société. Pour avoir une idée juste de la réalité sociale, il est nécessaire de comparer l'état de privation ou d'abondance des divers groupes humains qui constituaient la population du Canada. Deux critères complémentaires: la fonction politico et économico-sociale et la répartition géographique des individus, doivent servir de guide dans la détermination de ces différents groupements. Une première division, basée sur le critère de la fonction sociale, permet de distribuer la population en trois catégories: le peuple colonial, les militaires et les dirigeants. Les coloniaux, ouvriers et agriculteurs, for-

10 Lettres de Doreil, lettre du 28 avril 1758, RAPQ (1944-1945), 122. 1906), 442 .

11 Emile Salone, La Colonisation de la Nouvelle-France, (Paris, 
ment le groupe qui a eu le plus à souffrir de la situation. Non seulement ceux-ci manquent de vivres mais toute leur activité économique est enrayée:

Les peuples du Canada doivent naturellement être bien ennuyés de la guerre, plusieurs y ont péri, ils sont chargés des travaux les plus pénibles, ils n'ont point le temps d'augmenter leurs biens et même de rétablir leurs maisons, on leur a enlevé une partie de leur subsistance, plusieurs ont été sans pain pendant 3 mois, ils logent des troupes qui les incommodent, ils ne sont pas nourris pendant toute l'année, autant qu'ils croient en avoir besoin. ${ }^{12}$

Les coloniaux sont les premiers à être frappés par les mesures de l'autorité sur le rationnement des vivres. Dans les ordonnances, on lui préfère les militaires. À preuve, cette remarque de Montcalm: "Manque de vivres. Le peuple réduit à un quarteron de pain. Il faudra peut-être encore réduire la ration du soldat." 13 Dans les moments les plus difficiles, le soldat conservera sa demi-livre de pain tandis que le peuple devra se contenter de deux onces. ${ }^{14}$ D'ailleurs, ces deux onces de pain accordées au peuple étaient le résultat d'un compromis, d'un pis-aller. N'avait-on pas tenté, au mois de décembre, d'enlever entièrement le quarteron de pain ? Ce n'est qu'à la suite de fortes pressions qu'on était revenu sur cette décision. Quant à la viande de cheval, elle fut tout d'abord destinée aux coloniaux. "On a commencé aujourd'hui la distribution aux soldats de la chair de cheval... Il y a longtemps qu'on en distribue aux Acadiens et au peuple de Québec et de Montréal." ${ }^{15}$

Il semble que les coloniaux habitant les centres urbains ont souffert plus que les ruraux, des vicissitudes de la disette.

${ }^{12}$ La mission de M. Bougainville en France en 1758-1759, RAPQ (1923-1924), 28.

13 Lettre de Montcalm au Ministre de la Marine, 18 sept. 1757, dans Malartic, 157-158.

14 "Québec. Misère grande: le peuple réduit à deux onces de pain; le soldat à $1 / 2$ livre." Journal de Bougainville, 3 mai 1758, RAPQ (19231924), 319. 323.

15 Journal du Marquis de Montcalm, 9 décembre 1757, Casgrain, 7: 
Dans la plupart des cas, les descriptions de situations misérables concernent les villes de Québec et de Montréal. La population rurale peut vivre des produits de la ferme: viandes, légumes et laitage. Il lui est aussi plus facile de se soustraire aux ordonnances de l'administration. Par contre, dans les villes où la densité de la population est plus forte, trop forte même comparativement aux campagnes, ${ }^{16}$ la nourriture devient vite rare. De plus, le contrôle administratif étant plus efficace, les citadins sont forcés de se soumettre à la ration commune. Ainsi, à Québec, au cours de l'été 1757, "Monsieur l'intendant a différé de faire tuer les bestiaux jusqu'à ce que quatre cents quintaux de riz arrivés de Marseille soient consommés." ${ }^{17}$ En temps de crise économique, les populations urbaines sont toujours les plus désavantagées.

Pour ce qui est des militaires, il faut y distinguer la condition des soldats et celle des officiers. Les soldats n'ont pas subi le même traitement en raison de leur "hivernement", soit dans les garnisons des forts, dans les casernes des villes ou des villages ou chez les cultivateurs. Nous verrons plus loin que les soldats des forts sont ceux qui ont le moins souffert du manque de vivres. Par contre, ceux des villes ont été les plus défavorisés; ils furent, dans la plupart des cas, l'objet des ordonnances limitant les vivres. L'ordonnance du 16 octobre 1757 indique "qu'à commencer du ler du mois prochain, la ration seroit de livrer aux soldats des troupes de terre et de la marine qui tiennent garnison dans les villes ..."18 Ces derniers étaient dans une situation inférieure à ceux qui vivaient chez les ruraux. "Quelques difficultés que les troupes qui sont dans les côtes, éprouvent pour vivre chez l'habitant, leurs soldats seront encore moins à plaindre que ceux qui seront en garnison dans les villes." ${ }^{19}$ Dans une économie de subsistance, les soldats qui

16 "L'on a laissé les villes se peupler proportionnellement plus que les campagnes." Bougainville, Le texte des considérations sur l'état présent du Canada, Fascicule préparé par M. Roland Lamontagne pour les étudiants de la Faculté des Lettres de l'Université de Montréal, 3.

17 Journal du Marquis de Montcalm, 14 juillet 1757, Casgrain, 7: 243.

18 Vaudreuil à Lévis, 16 octobre 1757, Casgrain, 8: 46. (L'expression n'est pas soulignée dans le texte original.)

19 Montcalm à Lévis, 14 septembre 1757, Casgrain, 6: 54. 
séjournaient dans les campagnes, profitaient des avantages réservés aux agriculteurs. Quant aux officiers, la plupart ont donné l'exemple aux soldats dans l'application des règlements sur les vivres. Le 14 octobre 1757, Montcalm écrit à Lévis: "J'ai ouvert hier l'avis du retranchement des tables... J'ai été d'avis qu'il ne falloit, de tout l'hiver, ni bals, ni violons, ni fêtes, ni assemblées." 20 Ailleurs, il est dit: "Le peuple est au quarteron de pain. Ma maison et ceux qui ont dîne journellement chez moi sont au même ordinaire pour le pain." 21 Tous les officiers subalternes n'ont pas été fidèles à cette consigne. Quelques-uns, entraînés par des administrateurs, ont tenté de s'y soustraire. L'incident survenu au Fort Carillon est très significatif. "Une sédition avait éclaté au Fort Carillon à la suite des vols éhontés sur les subsistances militaires. Les soldats exaspérés avaient résolu de tuer leurs officiers et de livrer le fort aux Anglois." 22

De leur côté, les dirigeants coloniaux, administrateurs et grands commerçants, ne paraissaient pas inquiétés par la situation de la colonie. Si dans leur correspondance officielle, ils manifestent quelques inquiétudes, celles-ci ne transparaissent aucunement dans leur comportement. Tout l'hiver ne fut qu'une longue succession de festins et de bals. Alors que le peuple était réduit au quarteron de pain, l'intendant Bigot donnait un somptueux banquet où "il y a eu trois tables faisant quatrevingts couverts". ${ }^{23}$ A ces réceptions, les jeux de hasard étaient très à la mode et ils retenaient l'attention de la petite aristocratie. "On ne parle ici que de cent louis gagnés, perdus ... Les têtes sont totalement tournées. La nuit dernière, Mercier a perdu trois mille trois cents livres [Mercier était un des principaux fournisseurs de l'armée]." ${ }^{24}$

\section{ETAT DE CRISE}

Une telle situation, famine chez les uns et abondance chez les autres, ne pouvait que provoquer des critiques dans la popu-

20 Id. à $i d$., 14 octobre 1757, ibid., 6: 65.

21 Cité dans A. J. Malartic, op. cit., 160.

22 A. J. Malartic, op. cit. (note de l'éditeur), 159.

23 Journal de Montcalm, 18 décembre 1757, Casgrain, 7: 326.

24 Montcalm à Lévis, 26 décembre 1757, Casgrain, 6: 104. 
lation. Dans une lettre à la marquise de Saint-Véran, Montcalm écrit: "Il y a de la fermentation dans le peuple et les troupes." 25 Les soldats se regimbent. La plus célèbre révolte est survenue à Montréal le 1er novembre 1757. "On écrit de Montréal que le ler novembre, jour de la distribution des vivres sur le pied de la réduction, les soldats de la marine", ont refusé de se soumettre. ${ }^{26}$ Sans Lévis qui harangua ses grenadiers et les pria de donner l'exemple, des troubles sérieux auraient éclaté. Malgré les avertissements de Lévis, un état de tension a persisté pendant la majeure partie de l'hiver. "Par les nouvelles de Montréal, il y a toujours de la fermentation dans les esprits au sujet de la réduction de la ration et de la distribution de cheval." 27 Lorsque les troupes se révoltent, c'est très souvent sous l'influence des miliciens et des cultivateurs canadiens. "Le soldat [cantonné dans la région de Montréal] y est moins docile qu'à Québec parce qu'au lieu d'être caserné, il est logé chez l'habitant qui l'excite." ${ }^{28}$ Cette révolte des Canadiens contre l'autorité à propos des vivres est d'ailleurs alimentée par un phénomène d'ordre psychologique: le conflit habituel et nécessaire entre coloniaux et administrateurs métropolitains. Pour les coloniaux, les métropolitains sont des étrangers, des usurpateurs qui recherchent avant tout leurs intérêts personnels. "Le Canadien a un mauvais ton à cette occasion; il y est un peu excité par quelques curés; on se plaint nommément de celui de l'Assomption. Le grand mal vient de ce que le Canadien n'a pas de confiance dans le gouvernement, et reste toujours persuadé que la disette est artificielle et suggérée par l'avidité de certaines personnes." 29

Les femmes ont pris part à ce soulèvement contre l'autorité. A quelques reprises, elles se sont réunies pour manifester. Dans son journal, Lévis écrit que le ler décembre 1757 "il y eut une émeute de femmes; elles s'assemblèrent devant la porte de $M$.

25 Cité dans Malartic, op. cit., 160.

26 Journal de Montcalm, 6 novembre 1757, 7: 316.

27 Ibid., 14 décembre 1757, 324-325.

28 Ibid., 14 décembre 1757, 325.

29 Ibid., 6 novembre 1757, 316. 
le marquis de Vaudreuil; elles demandèrent à lui parler... Les femmes répondirent à $M$. de Vaudreuil qu'elles avoient de la répugnance à manger du cheval; qu'il étoit ami de l'homme; que la religion défendoit de les tuer et qu'elles aimeroient mieux mourir que d'en manger... Elles dirent qu'elles n'en prendroient pas, ni personne, pas même les troupes. Après quoi, elles se dissipèrent et se retirèrent chez elles en tenant des propos séditieux". ${ }^{30}$ Le 15 avril 1758, elles se réunissent encore, et cette fois, devant la porte de Monsieur Daine, lieutenant général de police. Dans une société où la majorité des hommes étaient affectés soit à la guerre, soit au transport des vivres, il était normal que les femmes prennent la relève. De plus, la question de la nourriture atteint directement la ménagère, la mère de famille. Dans ces cas, les femmes, d'ordinaire plus passives et plus conservatrices, n'hésitent pas à s'unir pour faire valoir leurs revendications. Quelques années plus tard, on rencontrera un phénomène identique dans l'histoire américaine: à la suite de la législation anglaise sur le thé, Samuel Adams réussit à soulever les femmes américaines contre l'impérialisme anglais.

\section{II — ESSAI D'EXPLICATION}

\section{LA CONJONCTURE POLITIQUE ET MILITAIRE}

A la suite de cette analyse des documents, une constatation s'impose: la question des vivres au cours de l'hiver 1757-1758 fut réellement un problème majeur pour le Canada. Mais une telle analyse descriptive, presque événementielle, est insuffisante pour expliquer l'origine et les causes de cette disette et pour comprendre toute la portée des inégalités sociales. Une étude exhaustive de cette question doit se situer dans le cadre de la guerre de Sept-Ans et tenir compte des données de base de la colonisation française en Amérique (relations métropolecolonie, relations intercoloniales, difficultés économiques...) ainsi que de l'état politique et économique du monde occidental, 118-119.

30 Journal du Chevalier de Lévis, ler décembre 1757, Casgrain, 1: 
à cette époque. Deux faits primordiaux déterminent la conjoncture politique et militaire du Canada: $1^{\circ}$ il est une colonie presqu'entièrement dépendante de la métropole française déjà affaiblie par la guerre en Europe; $2^{\circ}$ cette colonie doit défendre l'intégrité de son territoire contre l'expansion de colonies rivales.

En 1757-1758, le Canada n'en était pas à sa première disette. Au cours de la période de paix 1714-1744, la colonie avait été menacée de la famine à quelques reprises. Certes, entre celle-ci et celle de 1757-1758, on y trouve des caractéristiques semblables (mauvaise récolte, difficultés de ravitaillement) mais la situation qui a prévalu en 1757-1758 et durant la guerre de Sept-Ans se différencie des autres par la présence de facteurs nouveaux, entre autres, l'aspect déterminant de la guerre. Celleci est un agent de perturbation des structures économiques. Si elle peut profiter avantageusement à des pays non-engagés, elle risque, au contraire, de désorganiser la vie économique d'un pays lorsqu'elle se déroule à la frontière ou à l'intérieur de ce pays. Tout conflit militaire avec les colonies anglaises plaçait le Canada dans une position critique. Par suite de la faible densité de la population et de la vaste étendue de son territoire, il ne pouvait assurer la défense de ses frontières par ses seules ressources humaines et matérielles. Il devait compter presqu'exclusivement sur les troupes métropolitaines.

Dès lors, la guerre de Sept-Ans signifia pour le Canada: 1) un accroissement de la population; 2) une forte augmentation de la population des régions périphériques, la guerre se déroulant en grande partie sur les frontières éloignées. Ces conditions démographiques nouvelles et leurs interactions avec le facteur géographique posaient un double défi à la vie économique du Canada. D'une part, il était nécessaire d'augmenter la quantité des biens de consommation pour nourrir 10,000 nouvelles "bouches". D'autre part, il fallait assurer le ravitaillement en vivres des postes à la frontière. Pour autant qu'il saura répondre à ces nouveaux impératifs, le Canada pourra rétablir l'équilibre rompu par la guerre. 


\section{DÉMOGRAPHIE ET PRODUCTION}

C'est un fait que la guerre avait entraîné dans la colonie un accroissement de la population qui équivalait à une augmentation du nombre des consommateurs. On évaluait le nombre des immigrés à environ 9,000 à 10,000, ce qui représentait un accroissement de $17 \%$. Ceux-ci se composaient de militaires français, d'Acadiens réfugiés, d'Indiens et de prisonniers anglais. Le 22 octobre 1757, Doreil écrivait: "En Comprenant les accadiens (sic) Refugiés Laugmentation de sauvages les prisonniers anglois Il a eu Neuf a dix mille bouches de plus qu'a L'ordinaire a nourrir." 31

L'arrivée de bataillons français occasionnait presque toujours des fluctuations dans l'activité économique. De nouveaux consommateurs apparaissaient. La perspective de la rareté des vivres provoquait un phénomène d'inflation. Certains dirigeants en étaient conscients. Le Ministre de la Marine avait même conseillé au gouverneur de tenir secrète la venue prochaine des troupes de terre "pour prevenir la cherté qu'elles pourront occasionner dans les denrées et marchandises du pays". ${ }^{32}$ La venue des troupes causait des problèmes alimentaires si sérieux que, d'après Bougainville, même si la France avait été en mesure d'envoyer de plus puissants renforts, la colonie n'aurait peut-être pas été capable d'absorber ce nouveau flot d'immigrants: "Quel moyen de résister si nous n'avons pas 8 ou 10 mille hommes de troupes entretenues, mais malheureusement la colonie ne pourra les nourrir que dans les années les plus abondantes et il faudra encore de temps en temps des secours de vivres de France." ${ }^{3}$ On voit ici comment les facteurs démographiques et économiques sont intimement liés. Toute modification des données démographiques nécessite un changement des conditions économiques sinon il y a déséquilibre. "En reflechissant sur La Situation

${ }^{31}$ Lettres de Doreil, lettre du 22 octobre 1757, RAPQ (1944$1945), 114$.

32 Cité dans Guy Frégault, François Bigot, Administrateur français (Montréal, 1948), 2: 113. 1924), 28. 
actuelle de cette Colonie, Peut on Demander une augmentation de Bouches Il faut esperer que l'on sera plus attentif qu'on ne La ete cette Année a envoyer de puissans secours en vivres." 34

En plus de la présence des troupes françaises, la guerre rendait nécessaire celle des Sauvages. Certes, en temps de paix, la diplomatie des Français avec les Indiens avait toujours coûté cher au roi en présents et cadeaux de toutes sortes. En temps de guerre, cette alliance devenait indispensable. Aux raisons politiques et économiques venait s'ajouter un motif d'ordre militaire. Le Canada avait besoin de combattants, de défenseurs et il devait éviter à tout prix que cet imposant contingent de guerriers ne s'allie aux Anglais. Dès que ces indigènes devenaient, pour ainsi dire, mercenaires, il fallait assurer leur subsistance et leur équipement.

Le Roi donne beaucoup de présents aux Sauvages des Pays d'en Haut, cela coûte année commune 150,000 francs; [...] Ces dépenses sont encore fort au-dessous de celles que l'on fait en temps de guerre pour équiper, armer, nourrir, gratifier, donner des colliers tant à nos Sauvages domiciliés qu'à ceux du Pays d'en Haut. ${ }^{35}$

De plus, comme ceux-ci sont absents de leur tribu et qu'ils ne peuvent cultiver et chasser, qui nourrira leur famille sinon le roi? Ce n'est plus 2,000 mais 6,000 Indiens qu'il faut approvisionner. Il s'ensuit une très grande consommation.

La nécessité de se servir des Sauvages est une autre occasion de fausse consommation [...] Nos Sauvages domiciliés ne veulent pas aller en guerre à moins qu'on ne nourrisse leurs femmes et leurs enfants, de sorte que si vous avez 2,000 sauvages, il faut des vivres au moins pour $6,000 . .^{36}$

La colonie devait aussi accepter dans ses rangs des Acadiens. La guerre les avait chassés de leur lieu d'habitation. Plusieurs

${ }^{34}$ Lettres de Doreil, loc. cit., 114.

35 Bougainville, Mémoire sur l'état de la Nouvelle-France (1757), RAPQ (1923-1924), 66 .

${ }^{36}$ La mission de Bougainville en France en 1758-1759, loc. cit., 26-27. 
se réfugiaient dans la vallée du Saint-Laurent et principalement à Québec. Ce transfert de population atteignit son point maximum en 1757-1758. Environ 2,000 Acadiens étaient venus s'établir dans la colonie à cette date.

La colonie sert de refuge à un grand nombre d'Acadiens. Ces malheureux arrivent de partout. A Québec, il en débarque 200 en 1756,130 en 1757. Ces derniers sont des habitants de l'Ile Saint-Jean qui fuient la famine. D'autres viennent de l'isthme après la capitulation du fort Beauséjour. D'autres enfin s'évadent des colonies anglaises. En 1757, Montcalm fixe le total de ces réfugiés à 1800, Lévis a 2,000. En 1758, seulement à Québec, Bigot en signale de quinze à seize cents. ${ }^{37}$

Leur état lamentable ne pouvait qu'augmenter celui des Canadiens. Le Canada, déjà en proie à de multiples difficultés d'approvisionnement, devait prendre en charge et nourrir un nombre supplémentaire de personnes.

Cet accroissement remarquable et subit des consommateurs nécessitait une augmentation de la quantité des biens de consommation. La colonie a-t-elle su relever ce défi? Analysons les diverses sources d'approvisionnement: la production locale; les importations; les prises à l'ennemi et les ressources naturelles (économie de cueillette).

\section{- la production locale}

La production alimentaire du Canada paraît avoir connu une baisse au cours de l'année 1757. Deux facteurs tendent à l'expliquer: le manque de main-d'œuvre et les conditions climatiques défavorables. En 1757, l'agriculture n'a pas atteint le développement qu'on en avait espéré. La superficie cultivée des terres défrichées ne correspond pas à un cœfficient normal de mise en valeur des possibilités du sol. Des quelques milliers d'âcres disponibles, plusieurs demeurent en friche. En 1755, Vaudreuil écrivait à Machault: "Si les terres étoient cultivées, la colonie seroit en etat de nourrir autant de monde qu'il plai-

37 Emile Salone, op. cit., 447-448. 
rait au roi d'y en faire passer, mais les habitants sont épuisés; ceux qui depuis plusieurs années ont pris des terres, n'ont pu seulement les défricher." 38 Mises à part les prédictions ronflantes de Vaudreuil, nous retenons de ce témoignage qu'il y a perte dans l'utilisation du sol et que la cause de ce phénomène réside dans l'absence d'une main-d'œuvre adaptée.

Le manque d'une main-d'œuvre satisfaisante a été la pierre d'achoppement de la vie agricole canadienne. La multiplicité des tâches à remplir et l'immensité de l'espace à coloniser divisaient les forces des travailleurs. Affectés à différents travaux, ceux-ci n'accordaient pas la priorité à l'agriculture. Depuis les origines de la colonie, la vie de coureur de bois avait fourni à la jeunesse du pays de meilleurs attraits que celle de défricheur. Les jeunes quittaient les terres pour l'aventure. En 1757-1758, cette situation prévalait encore ${ }^{39}$, mais la pénurie d'agriculteurs était accentuée par la guerre qui sévissait. Tout cultivateur en état de porter les armes devait faire partie de la milice. Et "chaque levée de miliciens privait le sol d'autant d'ouvriers nécessaires". ${ }^{40}$ Du printemps à l'automne, ces hommes faisaient campagne avec les troupes régulières. D'autres étaient employés à mener les bateaux pour le transport des troupes et l'approvisionnement des armées à l'intérieur du pays. Les énormes distances à parcourir nécessitaient un grand nombre d'ouvriers. Tous, ils devaient abandonner leurs terres et leurs familles pour la défense de la colonie. Ce déplacement de la main-d'œuvre était d'autant plus néfaste pour l'agriculture qu'il avait lieu durant la saison où les travaux de la ferme requéraient le plus de travailleurs. En leur absence, les femmes, les vieillards et les enfants s'étaient occupés de cultiver les champs. Malgré leur bonne volonté, on ne pouvait attendre d'eux les meilleurs résultats. Lorsqu'à l'automne les miliciens retournaient dans leurs foyers pour les dernières récoltes, ceux-ci, épuisés et "affaiblis par la fatigue des marches et des navigations continuelles", ${ }^{41}$

38 Cité dans Guy Frégault, op. cit., 111.

39 Bougainville note que la traite "occupe au moins 2,000 hommes chaque année". Voir Bougainville, Le texte des considérations sur l'état présent du Canada (1758), loc. cit., 2.

40 Guy Frégault, op. cit., 2: 166 .

41 Bougainville, ibid., 2. 
ne pouvaient fournir un rendement aussi efficace qu'en temps normal. Bougainville était conscient de ces problèmes. Il avait même proposé un meilleur emploi des ressources humaines qui, tout en tenant compte des impératifs militaires, ne négligerait pas la production agricole.

La seule attention à avoir dans le moment présent, c'est d'être très sobre sur l'employ des hommes, d'éviter surtout les mouvemens inutiles, et pour ainsy dire les faux frais de fatigue, de régler les commendemens des miliciens avec égalité, justice et intelligence, de ne point faire marcher des enfans comme je l'ay vu pratiquer, afin de disposer autant que les circonstances et l'ennemi le permettront, les expéditions de guerre pour les tems, où la terre nexige pas la présence des habitants. ${ }^{42}$

En somme, si la guerre avait provoqué un accroissement subit du nombre des consommateurs, elle avait, par contre, contribué à diminuer le nombre des producteurs sans pour cela améliorer les moyens techniques de production agricole.

Quoique importante, l'influence de ce facteur démographique n'est pas suffisante pour expliquer la plus ou moins bonne récolte de 1757. À celui-ci s'ajoute des facteurs géographiques. Ceux-ci ont altéré la production des céréales et celle des animaux de boucherie.

Les conditions météorologiques au cours de l'année 1757 n'ont pas été favorables à la moisson. Le gel et la pluie ont endommagé la récolte des grains. Dans une lettre à Moras, Bigot décrit ainsi le pays:

Mais il est malheureux dans ses récoltes [...] il gèle et il pleut depuis 15. jours ou 3. semaines. Les bleds sont roüillés et échaudés [...] Si vous ne preniés pas, Monseigneur, des précautions pour assurer le départ des batimens chargés de vivres que le munitionnaire demande en France, la famine seroit plus affreuse l'année prochaine à Québec qu'elle ne l'a esté cette année. ${ }^{43}$

42 Bougainville, ibid., 2. 2: 226.

43 Bigot à Moras, 24 août 1757, cité dans Guy Frégault, op. cit., 
Vaudreuil tient aussi des propos semblables: "Les épies ne produisent presque rien, les pluyes continuelles que nous avons eues ont brûlé et rouillé nos grains." 44 De plus, la mauvaise qualité du grain occasionne des pertes lors de l'entreposage.

Pour ce qui est de la viande, la situation du Canada dans la partie septentrionale de l'Amérique empêchait l'élevage des bovins sur une grande échelle. Les longs hivers ainsi que la faible étendue des terres défrichées faisaient en sorte que l'habitant ne pouvait élever que quelques animaux. Bougainville nous livre, à ce sujet, des observations très justes:

Cette longueur de mauvais temps fait que les habitants ne peuvent élever d'animaux qu'autant ils auront du fourrage pour les nourrir pendant tout ce temps qu'ils sont obligés de les tenir dans les étables. De là vient que ce pays ne saurait jamais être abondant en viande de boucherie, surtout quand il y a consommation extraordinaire. ${ }^{45}$

Or, l'hiver 1757-1758 a été très long. ${ }^{46}$ Il est donc certain qu'il a eu des conséquences sur la consommation des céréales car l'on sait qu'en plus du fourrage, les bêtes à cornes ont besoin de moulées et de grains. "On a commencé le 3 [novembre] à aller en carriole. C'est de bonne heure et un surcroit de misère parce qu'il faut nourrir les bestiaux et les volailles qui ne trouvent plus de pâture à terre." 47 En plus d'exercer leur influence défavorable permanente, les conditions géographiques ont joué un rôle accidentel primordial qui a eu pour résultat d'abaisser la production et d'augmenter la consommation. Facteurs démographiques et géographiques semblent avoir produit un effet identique: une diminution de la quantité moyenne de vivres par habitant.

Des facteurs d'ordre politique et même psychologique ont agi dans le même sens et ont accru l'effet de ces derniers. Au

\footnotetext{
44 Vaudreuil à Moras, 12 septembre 1757, cité dans Guy Frégault, op. cit., $2: 227$.

45 Bougainville, Mémoire sur l'état de la Nouvelle-France (1757), RAPQ (1923-1924), 42.

46 Lettres de Doreil, lettre du 28 avril 1758, RAPQ (1944-1945), 122.

47 Journal de Bougainville, 3 novembre 1757, RAPQ (1923-1924), 314.
} 
printemps 1757, il était difficile pour l'agriculteur de se procurer des grains de semences. En effet, au cours de l'hiver précédent, les officiers de Bigot avaient perquisitionné toutes les fermes et avaient "acheté" au compte du roi tous les grains qui s'y trouvaient. Au printemps, les cultivateurs voulurent ensemencer et Bigot leur vendit des grains. ${ }^{48}$. Mais le prix d'achat élevé et la limitation de la quantité de blé à ensemencer obligèrent les fermiers à en semer une plus faible étendue. Ces mesures eurent une incidence malheureuse sur la récolte.

Quant à la production de la viande, une politique plus rationnelle d'élevage du bétail eut été nécessaire pour surmonter les difficultés naturelles issues du contexte géographique. $\mathrm{Au}$ Canada, les agriculteurs accordaient la priorité à l'élevage des chevaux. Les raisons en étaient simples: le cheval était l'animal que l'on préférait pour transporter les hommes et les marchandises et pour effectuer maints autres travaux. ${ }^{49}$ Pour les jeunes, le cheval était un objet de richesse et de convoitise et "chaque garçon qui a [avait] la force de manier le fouet a [avait] le sien". ${ }^{50}$ Pour un pays dont les ressources en fourrage et en blé étaient faibles, cette surproduction des chevaux était lourde de conséquences: elle empêchait l'élevage des bovins et des autres animaux de boucherie sur une plus grande échelle. Les chevaux consommaient les grains et le foin qui auraient pu être employés pour les bœufs, les porcs et les moutons. "Il faut remarquer qu'il n'y a point d'habitants qui n'aient plusieurs chevaux, [...], c'est ce qui empêche l'habitant d'élever autant de bœufs qu'il le ferait. Au lieu de trois ou quatre chevaux, il aurait dix à douze bêtes à cornes, et autre cela il pourrait élever plus de cochons, parce qu'il n'est point de garçon d'habitants qui ne vole son père pour donner de l'avoine

48 "Par lui-même ou par ses commis, il s'empara de tout le blé du gouvernement de Québec et Maurin et Penisseau de celui de Montréal. Ils en vendirent pour semer aux habitants à vingt-quatre livres le minot, eux à qui il ne coûtait que six livres." Mémoire du Canada, RAPQ (19241925), 134.

58.

49 Voir: La mission de Bougainville en France en 1758-1759, loc. cit., loc. cit., 42 . 
ou d'autres grains à son cheval, afin qu'il soit gras et vif." ${ }^{51}$ On comprend pourquoi durant l'hiver 1757-1758 la population canadienne fut réduite à manger du cheval. C'était "pour ne pas détruire entièrement l'espèce des bœufs et il [était] de l'intérêt politique de la colonie de diminuer celle des chevaux". ${ }^{22}$ L'élevage du mouton aurait pu jouer le rôle d'élément stabilisateur de la production de la viande. Par l'application d'une politique de régularisation des agnelages, les agriculteurs auraient pu accroître le nombre de leurs moutons sans pour cela augmenter la consommation des grains et du foin. Il eut été facile de réglementer l'accouplement du bélier et des brebis pour que celles-ci mettent bas en mai. Ainsi, les jeunes agneaux auraient trouvé leur pâture dans les champs et auraient pu être élevés "sans peine et sans frais". Mais les cultivateurs, peu préoccupés par ces problèmes, laissaient en tout temps les brebis avec le bélier. Il s'ensuivait que la naissance des agneaux en automne ou en hiver entraînait une plus forte consommation de foin et d'avoine et une élévation marquée du taux de mortalité: ce qui désintéressait les "habitants" de ce genre d'élevage. ${ }^{53}$

Certes, il est assez facile de voir l'action déterminante de ces facteurs démographiques, géographiques et politiques sur la production agricole du Canada, mais il est plus difficile d'en déterminer la portée exacte à l'intérieur d'un vaste ensemble de causes influençantes. Ainsi, par exemple, peut-on savoir

\footnotetext{
51 Bougainville, ibid., 42.

52 Journal de Montcalm, 9 décembre 1757, Casgrain, 7: 323.

53 "... une mauvaise pratique suivie par les habitans s'oppose à cette propagation [élevage du mouton] que la nature semble toute fois avoir voulu favoriser dans ce pays plus qu'en aucun autre. Puisque les brebis portent communément deux agneaux, comme on les laisse en tout temps avec le bélier un grand nombre mettent bas dans le mois de février, tems auquel la terre est couverte de neige. Il faut donc nourrir les agneaux dans les étables avec du foin et de l'avoine; il en périt la plus grande partie et d'ailleurs la cherté de cette nourriture dégoûte les habitans d'en élever. Un règlement qui ordonneroit de séparer le bélier dès le mois de septembre et de ne le laisser aprocher des brebis qu'en février, entreroit dans les vues de la nature, les agneaux nés en may, tems auquel la neige a fait place à la verdure, s'éleveroient sans peine et sans frais, il en périroit peu et le Canada fourniroit beaucoup de laine. [...] La chair du mouton seroit une ressource de nourriture des hommes qui ne trouvant pas toujours du bouf chés le boucher, mangent des chapons et des dindes l'hiver." Bougainville, Le texte des considérations sur l'état présent dw Canada (1758).
} 
d'une façon précise quelles ont été les répercussions de la mauvaise récolte sur la famine qui a sévi? Cette question est justifiée par le fait que dans les années 1730-1740, le Canada était devenu exportateur de blé. Des administrateurs comme Bigot et Vaudreuil n'auraient-il pas exagéré les conséquences de cette faible production, l'un pour voiler ses rapines et accumuler les profits sur les vivres provenant de France, l'autre pour cacher la faiblesse de son administration ? Le Mémoire du Canada tend à confirmer ce doute: "Si l'on eût ménagé ces comestibles, on aurait très aisément pu se passer des secours de France." 54 Pour élucider cette question, il faudrait inventorier minutieusement tous les documents existants et tâcher d'évaluer statistiquement quelle a été la production agricole du Canada au cours de la période concernée. On pourrait ensuite calculer la production moyenne de vivres par habitant. Le Mémoire $d u$ Canada affirme: "L'on fit des levées de blés dans tout le pays. La seule côte de Chambly, qui est une petite partie, en fournit trente mille minots." 55 Par contre, Bougainville rapporte que le 14 octobre 1757, à une réunion des dirigeants de la colonie, le munitionnaire avait exposé “qu'il n'avait que 1500 quarts de farine, que les recherches faites à la côte du Sud ne produiraient que 2000 quintaux, que le gouvernement de Montréal ne pourrait aider Québec que de $600 \ldots .{ }^{56}$ Ces affirmations nous paraissent contradictoires. Seule une recherche patiente pourrait jeter de la lumière sur toutes ces données. Pour évaluer la production de la viande, on se heurte aux mêmes difficultés. On sait qu'à l'automne 1757, il y avait dans la colonie 3000 chevaux que l'on pouvait abattre sans que la colonie en subisse des conséquences néfastes. Pour leur part, les bestiaux étaient rares, mais on ignore encore quel en était le nombre.

\section{- l'apport métropolitain}

Qu'une colonie ait de la difficulté à vivre par elle-même, c'est normal: la métropole est là qui veille au ravitaillement.

54 Mémoire du Canada, RAPQ (1924-1925), 135.

55 Ibidem, 135.

56 Journal de Bougainville, 14 octobre 1757, loc. cit., 311. 
Dans le cas du Canada, la métropole française a-t-elle rempli son rôle ? Fut-elle assez vigilante à secourir la colonie ou bien a-t-elle fait preuve de négligence ? Plusieurs historiens ont accusé la France d'avoir abandonné cette colonie à ses propres ressources. À plusieurs points de vue, ces accusations sont fondées. A la suite de fortes pressions de la part des philosophes et de l'aristocratie réactionnaire, le Ministère de la Marine avait dû limiter, au cours du XVIIIième siècle, les investissements dans les colonies et notamment au Canada. Ce pays n'avait pas été complètement délaissé par la métropole. Celle-ci avait continué à boucler partiellement les déficits et à fournir les articles nécessaires à la survivance de sa colonie, mais au lieu de suivre une courbe normale de progression, sa participation est demeurée dans l'ensemble plus ou moins stationnaire.

$\mathrm{Au}$ point de vue alimentaire, il semble qu'au cours de la guerre de Sept-Ans, les administrateurs français ont intensifié leurs efforts pour procurer aux habitants du Canada la subsistance nécessaire. Certes, au printemps et à l'été 1757 , le nombre des bateaux qui ont accosté au port de Québec est fort réduit. Dans son journal, Montcalm parle de trois bateaux arrivés à la mi-juin. Et par la suite, durant tout l'été, aucun navire n'a fait son apparition dans le fleuve. Ce n'est qu'au mois de septembre que les bateaux se présentèrent; le 9 septembre, le Saint-Patrice, avec 4000 quarts de farine; le 27, le Bristol, chargé de vins, d'eau-de-vie et de marchandises sèches. Au total, environ 15,000 quintaux de farine avait été destinés à la colonie au cours de la saison 1757. Pareille quantité de farine pouvait nourrir 60,000 personnes pendant 50 jours à raison d'une demi-livre de pain par jour. A cette ration, les 10,000 nouveaux consommateurs pouvaient être alimentés pendant 300 jours. Sans être extraordinaire, la contribution de la métropole était raisonnable. À ce sujet, Guy Frégault écrit:

En réalité, le ministre n'avait pas tellement tort de supposer le Canada au moins partiellement ravitaille. ${ }^{57}$

57 Guy Frégault, op. cit., $2: 231$. 
D'ailleurs, d'autres documents démontrent la volonté du ministre d'alimenter la colonie.

Le 10 février [1758], il annonçait que l'Etat expédiait 16,000 quintaux de farine, tandis que les correspondants de Cadet, Gradis à Bordeaux et Gazan à La Rochelle, préparaient des cargaisons exceptionnellement considérables de vivres pour le Canada. ${ }^{58}$

En août [1758], le ministre de la Marine résumait ainsi les divers efforts que la métropole poursuivait encore pour ravitailler la colonie; elle venait d'expédier 59,378 quintaux de farine, sans compter d'autres comestibles, comme du riz et du maïs. ${ }^{59}$

Mais ces vivres ont-ils atteint leur lieu de destination ? Les chiffres que nous possédons rendent compte des quantités de vivres expédiées par la métropole et non de celle reçues dans la colonie. Entre ces deux moments de l'expédition, la différence des quantités était grande. Celle-ci l'était d'autant plus que les inconvénients et les risques de perte dans le transport des vivres étaient accrus par la guerre de Sept-Ans. Certains facteurs géographiques avaient toujours existé mais combinés au facteur militaire, ils prenaient un relief plus accentué. À cause de la courte durée de la saison de la navigation sur le Saint-Laurent et de l'énorme distance entre la métropole et la colonie, les bateaux ne pouvaient pas transporter plus d'une cargaison par année. Et encore fallait-il qu'ils apportent avec eux une forte quantité de nourriture destinée à l'équipage. "Le séjour de ces vaisseaux ici nous était fort à charge; ils consommaient des vivres de toute espèce." ${ }^{0}$ Les tempêtes en haute mer et dans le Golfe provoquaient aussi le désastre de nombreux navires. "On dit aussi que le Foudroyant, navire du premier convoi, portant des recrues et tous les présens du Roi aux Sauvages, a fait naufrage sur la côte de Terre-Neuve." 61 Le facteur géographique prédominant semble avoir été la pré-

58 Ibidem, 236.

59 Ibidem, 238.

60 Journal de Bougainville, 16 septembre 1757, loc. cit., 308.

61 Ibidem, 3 juin 1758, 324. 
sence de nombreux écueils à l'entrée et dans le cours du SaintLaurent. A tout moment, on apprend qu'un navire a échoué. "[...] annonce que le navire le Lion d'Or, 1500 quarts farine, 400 qts de lard, est échoué à l'isle aux Lièvres". ${ }^{62}$

Ces difficultés étaient rendues plus amples par l'impréparation de la marine marchande française. On sait qu'au XVIIIième siècle, la France avait négligé de rebâtir sa marine marchande parce que pour des motifs d'ordre scientifique et culturel, on avait préféré construire et équiper des navires destinés plus spécialement à des missions scientifiques. En conséquence, on avait assisté à la décadence de cette marine marchande et de la marine de guerre: vieillissement de l'équipement, diminution de la main-d'œuvre spécialisée. En temps de paix, il n'y avait pas de problème pour le Canada. Les quelques convois qui faisaient la navette entre la France et la colonie suffisaient mais lorsque, au cours de la guerre de Sept-Ans, les besoins de la colonie nécessitèrent un plus grand nombre de navires, les plus lucides se posèrent la question: "La France a-t-elle des vaisseaux et des matelots? Assez pour les mers de l'Amérique et celles de l'Europe ?"' 63 Pouvait-on aventurer de vieux vaisseaux au milieu de l'Atlantique? Et ces bateaux, en nombre supplémentaire, pouvaient-ils se hasarder dans le fleuve avec des pilotes sans expérience? Encore de nos jours, les transatlantiques font appel à des pilotes spécialisés pour remonter et descendre le Saint-Laurent. La guerre était donc indirectement à l'origine de l'accroissement de ces difficultés. Elle allait en occasionner d'autres, et cette fois directement. La présence de la marine de guerre anglaise dans le golfe SaintLaurent à l'Île Royale et à l'embouchure du fleuve à Gaspé compliquait la situation. Plusieurs navires marchands furent interceptés au cours de la saison 1757. "Arrivée de la Judith. Nous donne de fâcheuses nouvelles du second convoi qui vraisemblablement a été pris presqu'entier." 64 Ceci avait d'autant

62 Ibidem, 30 mai 1758, 323.

${ }^{63}$ La mission de $M$. Bougainville en France en 1758-1759, RAPQ (1923-1924), 27.

64 Journal de Bougainville, 3 juin 1758, loc. cit., 324. 
plus d'importance que la prise d'un seul vaisseau pendant la guerre pouvait faire augmenter jusqu'à une valeur excessive le prix des marchandises dans la colonie. D'ailleurs, le site géographique rendait facile la capture des navires marchands. Les Anglais le savaient. Depuis 1629, ils employaient cette tactique. Bougainville avait proposé un plan pour remédier à la situation. Il projetait d'envoyer le ravitaillement par la Caroline ou bien de "faire escorter [les navires marchands] par des vaisseaux de ligne". ${ }^{65}$ La première de ces propositions lui apparaissait plus facile à réaliser.

A la suite de ces inconvénients, quel fut le volume de marchandises entrées dans le port de Québec au cours de la saison? La métropole calculait qu'environ les deux tiers des vivres expédiés se rendaient dans la colonie mais pour l'année 1757 et pour celles qui vont suivre, ce pourcentage est nettement trop élevé. Dans son journal, Bougainville note: "Dépêches du ministre de la marine; accuse la réception des lettres du 24 avril; nous croit dans une grande abondance de tout. À ce sujet, il y a une grande malversation dans ses bureaux." "66 L'éloignement de la colonie et la nécessité d'avoir recours à une foule d'intermédiaires causaient de nombreux problèmes administratifs. Ordinairement, les coloniaux devaient attendre jusqu'à deux ans avant de recevoir réponses à leurs demandes. Dans une période d'accélération de l'histoire, les solutions apportées par la métropole étaient souvent insuffisantes ou inefficaces car elles répondaient à des besoins qui n'étaient plus actuels ou bien à des impératifs largement dépassés par les circonstances nouvelles. Par suite de la guerre, la circulation maritime était devenue plus difficile et les risques de pertes plus grands. Il ne fallait plus se baser sur les estimations antérieures pour régler les exportations vers les colonies, mais dans la métropole, on ne semblait pas avoir pris conscience de ces éléments nouveaux. Une lettre de Doreil, datée du 25 octobre 1757, nous révèle que seulement 3 vaisseaux sur 18 avaient ravitaillé la colonie. Au total, au cours de la saison 1757, 51 navires étaient entrés dans le port

65 La mission de M. Bougainville en France en 1758-1759, loc. cit., 26. 66 Journal de Bougainville, 15 octobre 1757, loc. cit., 312. 
de Québec dont 17 pour le compte de l'entrepreneur. Parmi ceux-là, rares étaient ceux qui apportaient de la farine. Ils étaient surtout chargés de boisson et d'autres marchandises. Pour expliquer cette situation, Montcalm prétendait que Bigot lui-même avait détourné quelques bateaux vers Louisbourg et les Antilles. ${ }^{67}$ Ici comme pour la production agricole, un relevé de toutes les marchandises déchargées dans les ports de la colonie nous aiderait à évaluer la quantité exacte de vivres arrivés dans la colonie en 1757.

\section{— l'économie de cueillette et prises à l'ennemi}

La production agricole locale et les importations provenant de la métropole n'étaient pas les seules sources d'approvisionnement du Canada. Si au cours de l'année 1757, la guerre avait défavorisé le ravitaillement du Canada par la mer, elle l'avait néanmoins aidé sur terre. La prise du fort William Henry, au début d'août 1757, s'avéra une véritable aubaine. Les troupes françaises et coloniales $y$ trouvèrent en plus des munitions et des pièces d'artillerie, 1,237 quarts de lard pesant 230 livres et 1,737 quarts de farine d'environ 200 livres chacun. ${ }^{68}$ Quelques jours auparavant, on avait pris 100 bœufs, 150 moutons et 20 chevaux. Au mois d'avril 1758, les Canadiens capturèrent 200 bœufs. De telles prises devaient contribuer au bien-être de la colonie. Dans son journal, Bougainville écrivait:

Par un état exact des vivres actuellement dans les magazins de ce fort [Carillon: les vivres de William Henry y avaient été transportés], il y a en farine et lard de quoi nourrir les troupes qui y doivent tenir la campagne jusqu'à la fin d'octobre [d'après Bougainville, il y avait 8019 hommes concentrés dans cette région au mois d'août], la garnison qui y passera l'hiver, et un corps de 3000 hommes, si on veut $y$ en envoyer un au printemps jusqu'au 1er juillet. ${ }^{69}$

67 Journal de Montcalm, 2 juin 1759, Casgrain, 7: 535.

68 Journal de Bougainville, septembre 1757, 306.

69 Ibidem, 26 septembre 1757, 310. 
Dans son approvisionnement, le Canada pouvait compter sur une économie de cueillette. Les nombreux cours d'eau foisonnaient de poissons, les forêts, de bon gibier. Lorsque l'on manquait de vivres, on se livrait à la pêche et à la chasse. "Le Mis de Montcalm en réponse à leurs représentations, leur a envoyé des filets et des lignes pour qu'ils eussent à vivre de pêche et d'industrie. Voici le tems des tourtes, vraie manne pour ce pays; mais le plomb manque et l'on n'a pas sçu préparer du fer pour en tenir lieu." 70 Malartic signale ces sources de subsistance. Au cours de la campagne de William Henry, on a récolté une grande quantité de foin dans les clairières de la région du lac Champlain.

\section{GÉOGRAPHIE ET RAVITAILLEMENT}

Cette analyse des diverses sources d'approvisionnement ne nous permet pas de déterminer d'une façon précise si la quantité de biens de consommation a été suffisante ou non pour alimenter la colonie. Toutefois, exception faite de la prise de William Henry, nous pouvons affirmer avec certitude qu'un ensemble de causes défavorables a considérablement réduit la quantité de vivres attendue et tend à expliquer la situation de disette qui a prévalu dans la colonie, mais il est impuissant à rendre compte des inégalités sociales. Nous sommes alors en présence d'un problème de distribution des biens de consommation. Ce problème, causé en majeure partie par des facteurs militaires, démographiques et géographiques, fut réel et très important. Sa solution dépendait de l'attitude de l'administration coloniale et celle-ci, à partir de 1756 , se chargea de l'organisation du ravitaillement à l'intérieur de la colonie. Avant de voir comment les dirigeants coloniaux se sont acquittés de leur tâche, étudions les origines de ce problème.

\section{- la question militaire}

La défense de la colonie exigeait que les forces armées soient dirigées non pas au cœur de la vallée du Saint-Laurent

70 Ibidem, avril 1758, 319. 
mais aux limites du Canada. Au total, cela signifiait un accroissement d'environ dix mille nouveaux consommateurs aux frontières. Celles-ci étaient divisées en trois régions: les Grands Lacs, la Belle Rivière et le lac Champlain. Quand on songe que les forts de la région de l'Ohio étaient situés à plus de 500 ou 600 lieues du centre administratif, Québec, on voit toutes les implications de ce phénomène sur le ravitaillement. Avant la guerre, le problème était de moindre importance. Le nombre des soldats dans les garnisons des forts était sensiblement réduit. Plusieurs militaires s'établissaient avec leurs familles et pouvaient entreprendre la culture de quelques acres de terre. Ainsi, plusieurs établissements se suffisaient à eux-mêmes. On affirme que dans la région du Détroit les populations avaient un niveau de vie meilleur que celui des habitants de la vallée du SaintLaurent. Il est certain que, si au moment de la guerre, la population agricole au voisinage des forts avait été plus élevée qu'elle ne l'était, le problème du ravitaillement n'aurait pas pris les mêmes proportions. À l'accroissement de la population militaire (consommateurs) aurait dû correspondre l'accroissement de la population agricole (producteurs) mais tel ne fut pas le cas. Le Mémoire de Canada avait compris ce problème:

La cour aurait dû faire passer de sept à huit cents familles, ou davantage, au Détroit, Michilimakinac, et aux Illinois, des hommes propres à la culture des terres, leur concédant des terrains de même qu'à chaque famille; les aider dans cette entreprise, en leur donnant les ustensiles convenables à la culture; ... et dans peu d'années, on aurait pu sans difficulté y établir des greniers très riches, qui auraient été plus que suffisants pour l'entretien des garnisons des forts qu'on aurait voulu y bâtir. ${ }^{71}$

\section{- le facteur géographique}

Toutes ces suggestions sont demeurées à l'état d'hypothèse et il a fallu penser au ravitaillement. Or, toute question de ravitaillement est reliée à une question géographique. Selon leur portée, les facteurs de cet ordre peuvent avoir un double

${ }^{71}$ Mémoire du Canada, RAPQ (1924-1925), 125. 
effet: ils peuvent ou favoriser le commerce ou bien lui fournir des difficultés presque insurmontables. Au Canada, ceux-ci ont eu une influence négative. Des distances très grandes à franchir, des voies de communication naturelles semées d'embûches et des conditions climatiques particulières rendaient difficiles le transport des vivres et occasionnaient des pertes considérables. "La difficulté des transports en Canada occasionne une grande consommation de vivres en pure perte, et il est impossible d'y remédier." 72

Le facteur "distance" a une importance remarquable dans le commerce. Le problème de l'espace à franchir est indissociable de la dimension du temps. Plus la distance est considérable, plus le nombre d'heures pour effectuer le trajet est élevé. Dès lors, dans un pays comme le Canada où les forts étaient très éloignés des sources de ravitaillement, on ne pouvait pas faire plus d'un voyage par an. Un ravitaillement adéquat nécessitait la mise sur pied d'une armée de travailleurs affectés aux transports. Le Mémoire du Canada décrit ainsi la situation:

Ni M. de la Galissionnière [sic], ni M. Duquesne... . n'avaient fait attention à l'extrême difficulté d'y faire transporter les vivres et les effets nécessaires, tant par l'éloignement que par la difficulté des chemins [...] Il faut remarquer aussi qu'on ne fait qu'un seul voyage tous les ans... C'est une route de cinq à six cents lieues pour ces voyageurs. ${ }^{73}$

La lecture du journal de Malartic donne une idée très juste du grand nombre de personnes employées pour transporter les marchandises. Lorsqu'ils partaient pour les différents postes, tous ces gens devaient apporter avec eux leur subsistance pour la durée de l'expédition. Plus la distance à parcourir était grande, plus la consommation du travailleur était considérable. Ainsi entre la quantité de vivres expédiée au départ et la quantité arrivée dans les forts, il y avait souvent un écart prononcé.

Cet écart était aussi accentué par les mauvaises conditions de transport. Les routes carrossables entre les postes étaient

${ }^{72} \mathrm{~L}$ a mission de M. Bougainville en France en 1757-1758, loc. cit., 26. 73 Mémoire dw Canada, RAPQ (1924-1925), 124. 
rares. Les seules voies de communication étaient les cours d'eau. Bien souvent, ceux-ci n'étaient pas navigables sur tout leur parcours, d'où la nécessité des portages. Ces obstacles naturels forçaient les travailleurs à réduire leur cargaison qu'ils devaient transporter sur leurs épaules, à certains endroits.

Il faut considérer qu'il y a plusieurs portages à faire, à cause des chutes et des cascades qui se trouvent dans la route. Les farines et les pois ne peuvent être que dans des sacs pesant soixante livres... afin que les hommes qui conduisent ces voitures puissent en faire le portage commodément; car ils sont encore obligés de porter leurs canots avec tous leurs agrès et apparaux. ${ }^{74}$

Toutefois, si le canot était le moyen de transport le plus pratique, il n'en était pas pour autant le plus sûr. Il ne pouvait pas toujours résister aux intempéries et aux mauvaises conditions de navigation et assurer la conservation des vivres. Il en résulte souvent des pertes.

... les voitures dont on peut se servir sont des canots d'écorce ou de très petits bateaux, voitures peu solides, et encore moins propres à la conservation des vivres et des marchandises. Elles sont sans cesse exposées à la pluie et aux vagues des lacs et des rivières; aussi n'arrivent-elles la plupart du temps que pourries ou avariées. [...] Tantôt c'est un canot que les vagues ont jeté sur le rivage, ou submergé dans la rivière, ce qui entraîne pour le roi la perte des effets; tantôt une voie d'eau qui s'est déclarée pendant la traversée d'un lac, ce qui obligea de jeter les dits effets pour s'alléger, et conserver la vie aux voyageurs. Rien moins que tout cela le plus souvent. ${ }^{75}$

Portages, intempéries, naufrages et fuites d'eau occasionnaient donc des pertes continuelles qui réduisaient considérablement la quantité de vivres. Il faut aussi ajouter les fraudes et les friponneries qui se commettaient. Bien souvent, on vendait des marchandises à des commerçants rencontrés. "Pour

74 Ibidem, 124.

75 Ibidem, 124-125. 
remplir le vide de celle [eau de vie] qu'ils volent, ils ont recours à l'eau de la rivière ... Il s'agit seulement de remplir le vide d'une vente faite à quelque négociant rencontré en route, qui aura payé comptant, et donné des pelleteries en échange." 76

De plus, le ravitaillement était rendu plus difficile par la guerre. Les ennemis à l'affût guettaient le moment propice pour prendre ou détruire les cargaisons de vivres. Dans son journal, Malartic raconte un de ces incidents: “M. de Rouilly parti le 22 avec des traînes, escortées par quinze hommes pour aller chercher des vivres à Saint-Frédéric, a été attaqué à la presqu'isle par quatre-vingts Anglais." 77 La prise des forts où sont entassées les provisions causa des pertes considérables. Ainsi, à la remise de Chouaguen aux Anglais, et plus tard du fort Frontenac, les Français furent dans l'obligation de laisser entre les mains de l'ennemi de fortes quantités de vivres. Dans son journal, Bougainville analyse les répercussions d'une telle capture: "Il paraît aussi que tous les Sauvages de ces contrées [Niagara] gémissent de la destruction de Chouägen où ils trouvaient à bas prix les marchandises de traite, leurs besoins et leur superflu." 78

Devant ces innombrables difficultés à surmonter et ces risques permanents de perte, on s'attendrait à ce que l'administration coloniale ait négligé de ravitailler les forts de la frontière mais telle ne fut pas la réalité. En dépit de ces inconvénients, de grandes quantités de marchandises furent entreposées dans ces forts. La raison en est simple: tout le commerce intérieur de la colonie était monopolisé par un groupe de dirigeants politiques qui trouvaient très rentable d'expédier les vivres dans les postes éloignés.

\section{- l'organisation et l'administration du transport}

Depuis 1756, l'organisation du commerce intérieur dépendait uniquement d'un munitionnaire, Cadet, à qui l'administra-

76 Ibidem, 125.

77 A. J. Malartic, op. cit., 93.

78 Journal de Bougainville, RAPQ (1923-1924), 307. 
tion locale avait délégué ses pouvoirs. On avait confié à celui-ci le soin exclusif de l'approvisionnement et de la distribution des vivres pour toute la colonie. Cette organisation longuement méditée par Bigot et son ami Péan était née dans l'esprit d'un groupe d'administrateurs qui voulaient profiter de la situation provoquée par la guerre. Des conditions politico-militaires et démographiques nouvelles, insérées dans un cadre géographique spécifique, avaient fait naître des initiatives nouvelles. À ce sujet, Guy Frégault écrit:

Jusqu'à ce moment, le transport des vivres et des munitions de guerre dans les postes s'était effectué par économie, c'est-à-dire sans intermédiaire entre l'Etat et les particuliers chargés du travail. Les hostilités commencées tout de suite sur une grande échelle exigeaient des garnisons plus nombreuses dans les postes éloignés et, par conséquent, des provisions plus considérables. L'opération devenait donc plus intéressante qu'autrefois. Aussi vit-on surgir un entrepreneur. Qui était-il ? Une société, répond Varin. ${ }^{79}$

En elle-même, la guerre fournissait une conjoncture favorable pour la réalisation des désirs d'un petit groupe de profiteurs. Ne pouvait-elle pas servir d'heureux prétexte à toutes les mesures? Par la suite, dans sa défense devant la commission Duchatelet, Bigot se justifia à quelques reprises par cet argษment: tout ce qu'il avait fait, il l'avait accompli pour le plus grand bien de l'armée et la défense du pays.

Dès le printemps 1757, Montcalm décrit l'activité de cette société: "Une compagnie très protégée, qui, vraisemblablement sous des noms différents, fait le commerce exclusif du Canada."80 D'ailleurs, les termes de l'entente survenue entre les administrateurs et Cadet étaient non équivoques. L'entrepreneur s'engageait à fournir les vivres et les rafraîchissements nécessaires pour le service du Roi tant dans les villes de Québec, Trois-Rivières et Montréal, dans les campagnes où il y aura des troupes en quartier, à Lachine, que dans les postes du

79 Guy Frégault, op. cit., 2: 136.

80 Cité par Guy Frégault, op. cit., 2: 195. 
fort Frontenac, La Présentation, Saint-Régis, Niagara, Toronto, Saint-Frédéric, Carillon, Rivière Oyo et Gaspé. À la livraison des marchandises dans ces lieux, les garde-magasins remettaient à Cadet des reçus que celui-ci devait présenter à l'intendant pour le paiement. De plus, le prix des rations variait selon que celles-ci étaient fournies dans les villes, dans les campagnes ou dans les postes: fixé à 9 sous dans les villes, il s'élevait à 12 sous dans les campagnes et à 23 sous dans les postes. C'était un véritable monopole et de pareilles clauses ne pouvaient qu'exciter l'avidité de l'entrepreneur et de ses amis.

Selon la commission Duchatelet, il semble hors de tout doute que dans les seules années 1757-1758, l'intendant et son entourage aient réalisé un gain de douze millions sur des fournitures qui leur en avaient coûté onze. Pour accumuler de tels profits, les administrateurs avaient dû, d'une part, falsifier les divers états de compte en achetant les garde-magasins et d'autre part, causer l'inflation en provoquant artificiellement la rareté des vivres. Le premier genre de tactiques employées n'a pas eu ou peu d'influence sur le manque de vivres mais le second a contribué dans une large mesure à accentuer la disette. Pour réduire la quantité de vivres, deux méthodes étaient couramment utilisées: l'entreposage secret des vivres à certains endroits dans la Vallée du Saint-Laurent et l'expédition de fortes quantités de vivres dans les forts de la frontière. La connaissance de ces façons de procéder nous aide à comprendre en partie les inégalités sociales: l'une explique le traitement des soldats dans les forts de la frontière et l'autre, l'abondance de la classe administrative.

Il ne semble pas, en effet, que les forts éloignés aient été négligés. Au contraire, le munitionnaire a déployé ses efforts pour ravitailler les postes. La quantité de vivres trouvée par les Anglais au fort Frontenac en $\mathbf{1 7 5 8}$ jette de la lumière sur la politique des administrateurs. "Le colonel anglais Bradstreet... y trouva pour deux millions de marchandises, deux milles quarts de farine et cinq cents quarts de lard." 11 Malartic s'exprime

81 Cité dans A. J. Malartic, op. cit., 200. 
ainsi : "Les Anglais ... travaillent à embarquer les vivres, les munitions, l'artillerie et les marchandises, qu'ils ont trouvées en grande quantité." 82 On constate un déséquilibre quand on compare ces chiffres au nombre de personnes résidant dans ce fort. Au moment de la prise, en pleine campagne, il ne se trouvait que 70 hommes dans le fort.

De plus, cette politique d'entasser des vivres dans les forts à l'automne était d'autant plus contradictoire que la majorité des soldats passait l'hiver cantonnée dans la Vallée du SaintLaurent. Pourquoi accumuler des quantités énormes de vivres dans les postes quand on savait que seuls quelques soldats formaient la garnison durant la saison morte? Sous le couvert qu'il fallait avant tout assurer la défense du pays, on cachait le véritable motif. Celui-ci résidait dans le fait que l'expédition des vivres vers les forts rapportait beaucoup plus que le simple approvisionnement des paysans et des citadins. Le munitionnaire n'avait-il pas 14 sous de plus par ration pour les marchandises livrées dans les postes. On comprend pourquoi le munitionnaire avait insisté pour que Lachine soit considéré comme un poste des frontières, et qu'effectivement de grandes quantités de vivres y furent entreposées. Voilà qui explique pourquoi la famine fut plus intense à Québec et dans la Vallée du Saint-Laurent que dans les postes de la frontière.

Si cette pratique permettait à l'entrepreneur de réaliser de meilleurs gains, elle réduisait aussi la quantité de vivres par habitant à l'intérieur de la colonie. Ce n'était d'ailleurs pas le seul moyen employé. Pour provoquer une dimunition des biens et l'inflation, on avait recours à l'entreposage. C'était une pratique courante en temps de guerre dans tout le monde capitaliste occidental. $\AA$ Marseille, par exemple, on remarque un phénomène identique.

De 1756 à 1760: ils (les registres du port) nous signalent pour les années 1756-57, une stagnation des dépôts: le blé continue à arriver en dépit de la guerre [...] mais [...] il est clair que les négociants marseillais conservent, en dépôt, le blé qu'ils

${ }^{82}$ A. J. Malartic, op. cit., 201. 
parviennent alors à se procurer, dans la conviction où ils sont que du fait des hostilités en cours, les prix augmentent un jour ou l'autre. ${ }^{83}$

A Québec, on employait la même tactique. Si le peuple était dépourvu, l'administration recherchait ses intérêts et favorisait ses amis. Ainsi, le moulin de Péan à St-Michel regorgeait de farine. Dans l'île d'Orléans, on accumulait les réserves en cachette. La "Grande Société" se trouvait à l'abri de toutes les vicissitudes. Dans son journal, le 2 juin 1759, Montcalm s'exprime en ces termes:

On y trouve vingt milles minots de blé. S'il y en a partout ailleurs en proportion, pourquoi, depuis deux ans, le peuple meurt-il de faim et sommes-nous réduits à quatre et quelquefois à deux onces de pain ?... On a voulu le taxer [le blé] à bas prix, se charger de nourrir le peuple à bon marché, le faire payer cher au Roi, et on a mieux aimé cacher le grain que de le vendre. ${ }^{84}$

Alors que le peuple colonial avait souffert de la famine, la classe administrative avait vécu dans l'abondance. À un même défi lancé par le milieu, la réponse de l'homme avait été différente. Un premier groupe, l'infime minorité de la classe dirigeante, avait su profiter au maximum des moindres avantages de la situation tandis que l'autre en avait subi les contrecoups inévitables. Certains, à la suite de circonstances particulièrement favorables et grâce aussi à leurs initiatives personnelles, ont réussi à surmonter les difficultés et même à agir sur le milieu physique et humain dans lequel ils vivaient; d'autres moins favorisés ont été dépassés et subjugués par les événements. Un ensemble de facteurs géographiques, démographiques, politiques et militaires avait provoqué la disette, c'est-à-dire une diminution anormale de la quantité moyenne de vivres par habitant, mais cette diminution ne fut pas partagée également par tous les habitants du Canada. Cette disette n'était pas la résultante de l'action d'une série de causes exerçant leur influen-

\footnotetext{
${ }^{83}$ R. Romano, Commerce et Prix du blé à Marseille au XVIIIième siècle (A. Colin, 1956).

84 Journal de Montcalm, 12 juin 1759, Casgrain, 7: 534-535.
} 
ce isolément et indépendamment les unes les autres mais l'aboutissement de l'interaction d'un ensemble de facteurs. Ainsi, par exemple, nous avons pu constater que l'influence du facteur climatique sur l'élevage des bovins eut été atténuée par une politique plus rationnelle d'élevage du bétail; que, sans les difficultés de commerce entre la France et le Canada, difficultés accrues par la guerre, sans l'accroissement subit du nombre des consommateurs et la diminution du nombre des producteurs, la mauvaise récolte de 1757 n'eut pas joué un rôle aussi déterminant ... etc. En somme, nous pouvons affirmer que le problème des vivres au Canada au cours de l'hiver 1757-1758 n'est pas uniquement la conséquence d'une mauvaise récolte ou de la négligence de la métropole, mais qu'il est déterminé par la conjoncture militaire et politique du Canada, par des conditions géographiques et démographiques, tant permanentes (site, climat, densité de population) qu'accidentelles (accroissement subit de la population, pluies continuelles) et par des motifs psychologiques et sociaux.

Gilles ARCha'mbault, Département d'histoire, Université de Montréal 